\title{
summary
}

\section{Minocycline gel gives adjunctive improvement to scale and polish}

van Steenberghe $D$, Rosling B, Soder P-O, van der Velden U, Timmerman MFT, McCarthy EF, et al. A 15-month evaluation of the effects of repeated subgingival minocycline in chronic adult periodontitis. $J$ Periodont 1999; 70:657667

Objective To establish the efficacy and safety of $25 \%$ minocycline slow-release ointment when used intermittently as an adjunct to subgingival debridement in non-surgical treatment of routine chronic adult periodontitis over a prolonged period.

Design Multicentre double-blind randomised controlled trial at six university centres from 1993-1999.

Intervention Adults (104 in number) of 35-64 years of age, with at least one pocket $\geqslant 5 \mathrm{~mm}$ with attachment loss $\geqslant 3 \mathrm{~mm}$, radiographic evidence of alveolar bone loss in at least one site per quadrant, gingival index $\geqslant 1$ and plaque index $\geqslant 2$, were randomised to treatment with minocycline or a vehicle control. Patients using anti-inflammatory drugs or mouthwash and receiving antibiotics in the previous 3 months were excluded.

Outcome measures Probing depth, probing attachment level, sulcus bleeding index, gingival index, plaque index, $\%$ of sites improving $>2$ $\mathrm{mm}$ and microbial evaluation.

Results Eleven cases were excluded. Results showed significant reductions in micro-organisms measured, mean probing depth, bleeding index and gains in attachment. No hypersensitivity was reported, and $15 \%$ of the minocycline group and $27 \%$ of control group reported minor adverse symptoms.

\begin{tabular}{lcccc}
\hline \multirow{3}{*}{$\begin{array}{l}\text { Baseline } \\
\text { probing } \\
\text { depth }\end{array}$} & \multicolumn{2}{c}{$\begin{array}{c}\text { Sites demonstrating improvement } \\
>2 \mathrm{~mm}(\%)\end{array}$} & & \\
\cline { 2 - 3 } & Minocycline & Control & ARR & NNT \\
\hline $5 \mathrm{~mm}$ & 63 & 43 & 20 & 5 \\
$7 \mathrm{~mm}$ & 73 & 53 & 20 & 5 \\
\hline
\end{tabular}

Conclusion Repeated administration of minocycline ointment as an adjunct to scaling and polishing resulted in statistically significant improvements (over 15 months) in periodontal clinical status, and significant reductions in periodonto-pathogens for both therapies

Address for reprints: Dr D van Steenberghe, Department of Periodontology, Catholic University of Leuven, Kapucijnenvoer, B-3000 Leuven, Belgium.

\section{Commentary}

This well-constructed and well-executed study provides object lessons in clinical and statistical significance, clinical value and the use of microbiology. The results unequivocally demonstrate that minocycline gel provided a statistically significant improvement compared with controls. In both groups, initial mean pocket depths were $\sim 6.4 \mathrm{~mm}$. After treatment the mean pocket depths were approx. 4.6 and approx. $5.1 \mathrm{~mm}(P=0.0001)$ for the minocycline and the control group, respectively.

A $0.5-\mathrm{mm}$ difference is difficult for a clinician let alone a patient to discriminate, however, and the clinical value is also difficult to determine. To obtain these results, all patients were required to have scaling at baseline, 6 and 12 months, a reasonable clinical protocol.
In addition, the application of the gel required visits at 2 weeks, and at months 1, 3 and 9. Thus, by extrapolation to a clinical situation, to obtain the observed results a patient would need to make four additional trips to the dentist. They would also encounter additional fees.

The NNT of 5 makes the value point even more clearly. For every five sites treated with minocycline gel, only one would be expected to be significantly better than the control. Thus, the patient expends four additional trips and fees to treat five sites, so that one site will improve more than it would with scaling alone. In other words, out of the five treated sites, only one would improve by $0.5 \mathrm{~mm}$.

The microbiology results provide a third perspective. Periodontal disease is an infection and, presumably, if the pathogens are removed the risk of recurrence is significantly reduced. At the beginning of the experiment about $35 \%$ of sites had no detectable Porphyromonas gingivalis, a key periodontal pathogen. At 15 months, $74 \%$ of the minocycline group and $64 \%$ of the control group were pathogen-free. This suggests that $10 \%$ of sites in the control group might be more prone to recurrence. The potential value of this benefit has yet to be defined.

In summary, this clinical trial demonstrates efficacy, but at a level that makes me unlikely to prescribe it for patients.

Richard Niederman
Office of Evidence-Based Dentistry,
Harvard University and Forsyth
Institute, Boston, Massachusetts, USA

